\title{
Case Report: Ulcerative Colitis with Multiple Dural Venous Thrombosis
}

\author{
Safia Mohamud ${ }^{a}$ Mosunmola Oyawusi ${ }^{a}$ Roger Weir ${ }^{a}$ Richard M. Millis ${ }^{b}$ \\ Ozra Dehkordia \\ aDepartment of Neurology, Howard University Hospital, Washington, DC, USA; \\ bDepartment of Pathophysiology, College of Medicine, American University of Antigua, \\ Osbourn, Antigua
}

\section{Keywords}

Cerebral sinus vein thrombosis - Ulcerative colitis · Magnetic resonance venography

\section{Abstract \\ Background}

Cerebral sinus vein thrombosis (CVT) is a rare but serious complication associated with ulcerative colitis (UC), an idiopathic autoimmune inflammatory disease of the gastrointestinal tract. Management approaches for CVT remain unclear but may include anticoagulation and surgical thrombectomy. Herein, we report a case of a 23-year-old male who developed CVT with a history of UC. The patient was presented to Howard University Hospital when he slipped and fell. On arrival at the hospital, he complained of a headache with an aching sensation, associated with light/sound sensitivity. The patient had a history of uncontrolled UC. He had positive bloody diarrhea, lower abdominal pain, but denied any other neurological deficit. Computed tomography of the head showed left frontoparietal lobe hypodensities. Neurological exam was nonfocal. Vital signs were within normal range, but the patient experienced some memory loss and personality changes. Subsequent diagnosis of CVT was made with magnetic resonance angiography and magnetic resonance venography. Immediate treatment with low-molecular-weight intravenous heparin $(18 \mathrm{lU} / \mathrm{kg})$ was introduced. His UC was managed with methylprednisolone (60 mg IV daily), proton pump inhibitors, mesalamine, ciprofloxacin, and metronidazole. His condition gradually improved. On discharge, he was prescribed prednisone, azathioprine for his UC, levetiracetam for seizure, and warfarin with an INR goal of 2-3. In conclusion, the sudden onset and/or acute worsening of neurological status such as headache and mental confusion in a patient with UC should alert the treating physician about the possibility of CVT so that timely intervention could be employed to prevent disabling and potentially lethal sequelae of this disease.

\section{Karger"}




\section{Introduction}

Ulcerative colitis (UC) is a chronic inflammatory bowel disease (IBD) that is associated with a range of intestinal and extraintestinal manifestations [1-5]. Of these, dural sinus cerebral venous thrombosis (CVT) is a rare, potentially fatal extraintestinal manifestation of UC with only a handful of cases reported in the literature [6-8]. Clinical manifestations of CVT are variable and include headaches, vomiting, seizures, focal neurologic deficit, decreased level of consciousness, and visual impairment [9-12]. A large proportion of affected patients may have permanent neurological deficits or may die if not appropriately treated. Here, we describe the clinical presentation and treatment of a young-adult man with CVT associated with uncontrolled UC.

\section{Case Report}

A 23-year-old right-handed male with a 7-8 year history of uncontrolled UC presented to the emergency department of Howard University Hospital. On arrival, he complained of severe headache which he rated $8 / 10$ for intensity. The patient stated that 2 weeks earlier, he slipped and fell while taking a shower. After the fall, he stayed home to rest but did not think that the fall was serious enough to seek immediate medical attention. Since the fall, he experienced a holocephalic headache, which was getting worse over time. He described the headache as an aching sensation associated with light/sound sensitivity and nausea as well as generalized weakness. Since the fall, he also had experienced some memory loss. Other symptoms included positive bloody diarrhea, lower abdominal pain, slight dizziness, and headache, but he denied experiencing vision or hearing changes, numbness/tingling, or gait impairment. Abnormal computed tomography (CT) scan of the head, which showed left frontoparietal regions of hypo/hyper densities (Fig. 1), prompted neurological consultation. The neurological exam was nonfocal. Vital signs were within normal range, but the patient experienced personality changes and was irritable while interacting with the health-care team. During first week of admission, the patient also developed focal seizure. He was admitted to the neurology service and multiple teams including gastroenterology, infectious disease, and hematology were consulted for differential diagnosis.

Fig. 1. CT head without contrast. Left frontal lobe regions of decreased attenuation suggestive of vasogenic edema and left frontal lobe increased attenuation suggestive of intracranial hemorrhage. CT, computed tomography.

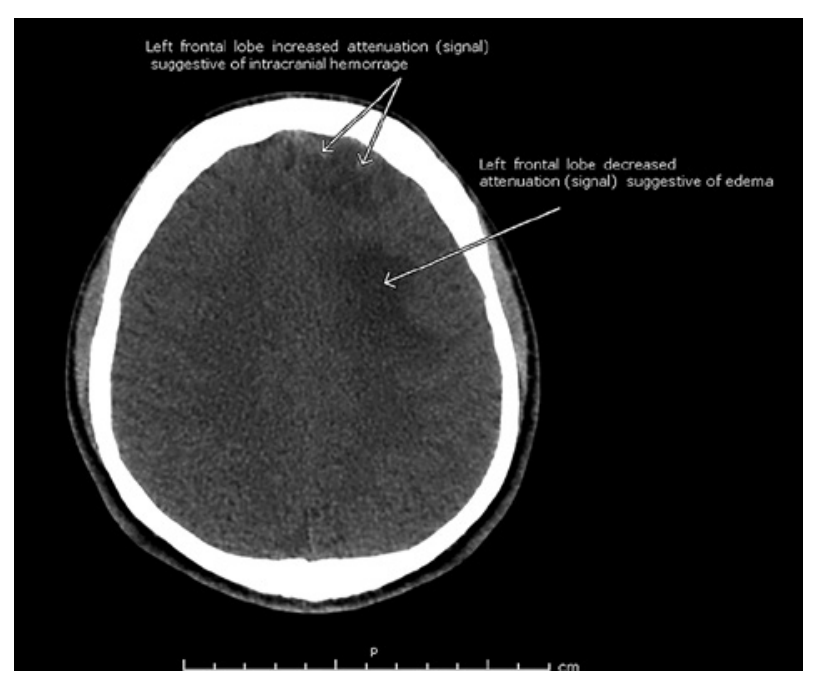



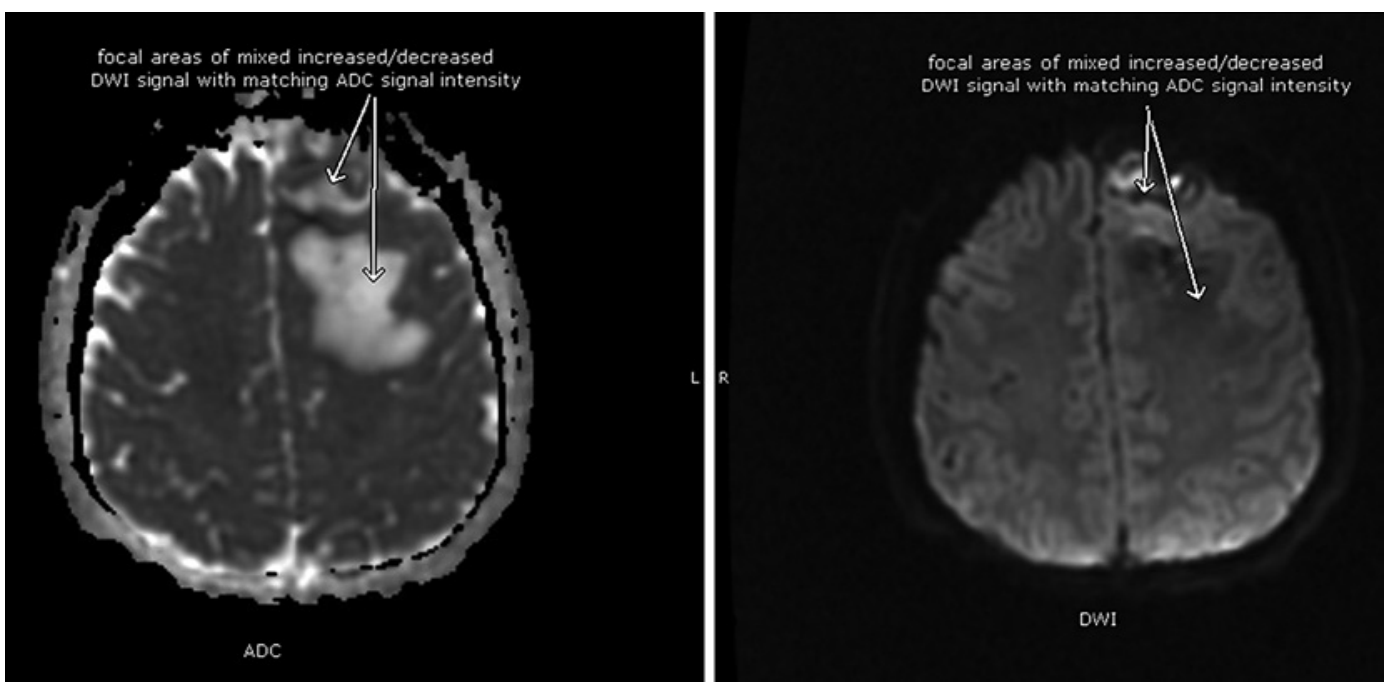

Fig. 2. MRI of the brain. Focal areas of mixed increased/decreased DWI signal with matching ADC signal intensity.

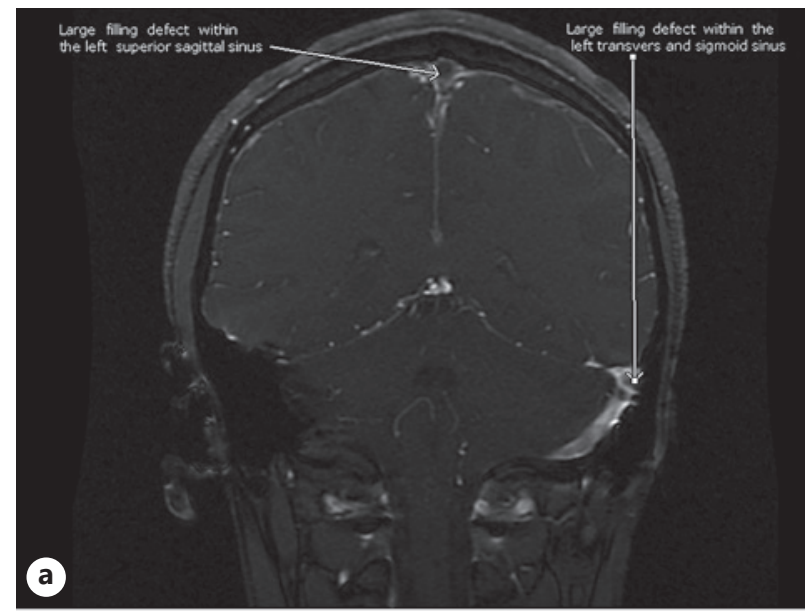

Fig. 3. MRV. a Coronal section showing superior and sigmoid sinus thrombosis (2 arrows). b MRV showing bilateral venous thrombosis involving proximal jugular vein and sigmoid sinuses. MRV, magnetic resonance venography.

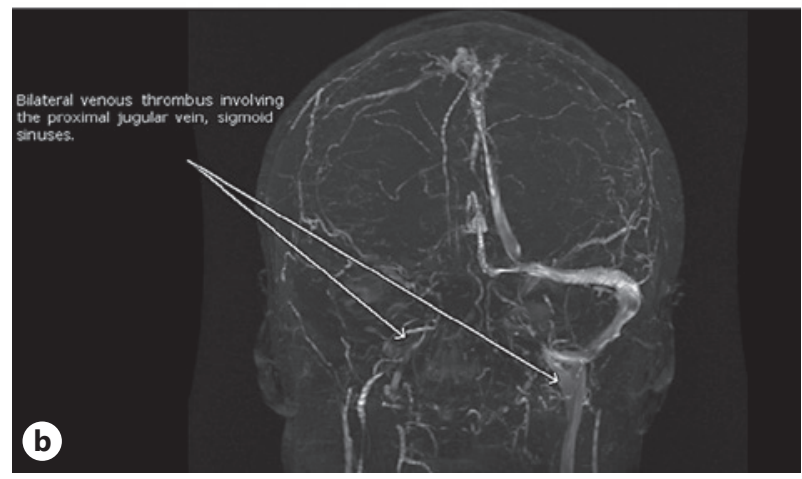

MRI of the brain showed focal areas of mixed increased/decreased DWI signal with matching ADC signal intensity (Fig. 2). Magnetic resonance angiography/magnetic resonance venography (MRV) showed dural venous sinus thrombosis in the bilateral sigmoid sinuses and the superior sagittal sinus, but there was no significant stenosis or occlusion of the intracranial arterial circulation (Fig. 3). The patient was transferred to the neurosurgery service 
Fig. 4. CT scan of abdomen and pelvis with contrast. Arrows show diffuse colonic wall thickening involving the mid-transverse colon to the rectum/anus compatible with significant inflammation in a 23-year-old man with UC. UC, ulcerative colitis; CT, computed tomography.

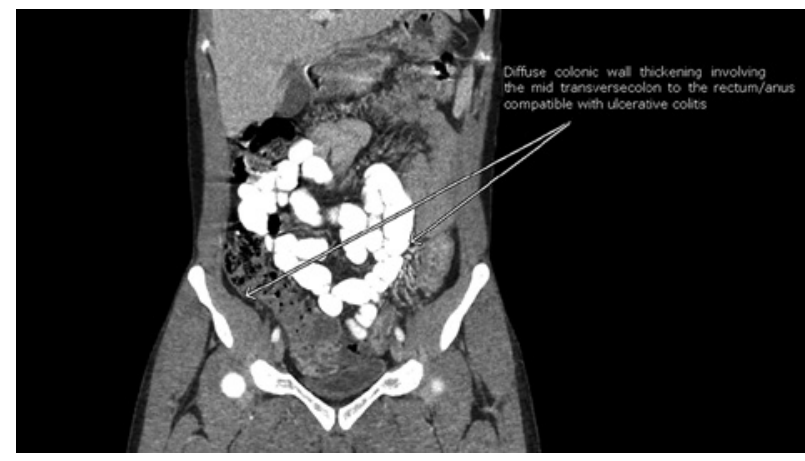

and the neuro-intensive care unit for further treatment and possible retrieval of the clot. Lowmolecular-weight heparin drip (18 IU $/ \mathrm{kg}$ ) was started immediately, and it was determined that no acute intervention was necessary at the time and the patient might only need surveillance MRV as an outpatient.

The gastroenterologist recommended CT scan of the abdomen, which showed diffuse colonic wall thickening extending from mid-transverse colon to the rectum/anus (Fig. 4) compatible with significant inflammation seen in patients with known UC. Sigmoidoscopy confirmed the presence of severe UC flare. Other laboratory testing included stool analysis with culture, white blood cell count, fecal calprotectin, Clostridium difficile, and C-reactive protein. Infectious diseases workup included Clostridium difficile, HIV, HSV serology, VDRL, toxoplasma IGM and IgG, John Cunningham virus (JC virus PCR), Cryptococci antigen, Fungal Ag panel, HSV, HBV Ag, T-Spot Tuberculosis Serum Galactomannan, Serum Beta Glucagon, ESR, procalcitonin, workup for rheumatoid arthritis, and antinuclear antibody. Hematological workup included Protein C and S, factor VIII, antithrombin III, and anticardiolipin antibodies. The majority of lab results came back within normal ranges with the exception of the inflammatory markers C-reactive protein, white blood cell count, ESR, and procalcitonin which were elevated due to uncontrolled UC.

The patient's UC flare was managed with fluid resuscitation and methylprednisolone (60 mg IV daily), proton pump inhibitors, mesalamine, ciprofloxacin, and metronidazole. He was also placed on levetiracetam for focal seizures, which were observed at admission. The patient was monitored daily to prevent increased intracranial pressure, expansion of hemorrhage by clinic examination, and head CT as needed.

\section{Discussion}

Multiple clinical studies have reported that patients with IBDs have an increased risk of venous thrombosis, including deep venous thrombosis, pulmonary emboli, portal vein thrombosis, Budd Chiari syndrome, retinal vein thrombosis, and CVT [1-6]. Among these, CVT is a rare complication of IBDs. It occurs in younger individuals during flares of the disease and appears to be more common in UC than in Crohn's disease [13]. CVT has multiple causes and variable clinical manifestations, making the clinical diagnosis and treatment extremely difficult.

Headache is the most common manifestation of CVT and is observed, to varying degrees, in the vast majority of patients $[9,14,15]$. In most patients, the headaches are acute and increase in intensity over days and weeks and may increase the index of suspicion with mimicry of the type of severe headache associated with subarachnoid hemorrhage and increased intracranial pressure. 
Focal neurological deficits including motor/sensory impairment, aphasia, cranial nerve palsy, and visual field disturbances and cortical blindness are observed in 18-72\% of CVT cases $[11,16,17]$. Seizures are seen in about $40 \%$ of patients $[10,18,19]$. In nearly half of the cases, seizure remains focal but can also generalize and even lead to status epilepticus [20]. Altered level of consciousness is another feature of CVT that has been reported in 14-18\% of cases [11, 21].

The clinical presentation of CVT depends on various factors such as location, extension of the thrombosis, the extent of the venous occlusion, and the nature of the underlying disease [22]. In the case presented herein, the patient had a history of uncontrolled UC and the symptoms at the time of presentation were mainly limited to a headache, memory loss, and personality changes. Other symptoms included bloody diarrhea, lower abdominal pain, and slight dizziness. CT scan of the head showed left frontoparietal lobe regions of hypo/ hyper densities suggestive of vasogenic edema and intracranial hemorrhage. Once the magnetic resonance angiography and MRV confirmed the presence of thrombosis in the superior and sigmoid sinuses, immediate anticoagulation therapy was initiated. The patient was also placed on levetiracetam for focal seizures, which were observed during admission. CT scan of the abdomen and sigmoidoscopy confirmed the diagnosis of UC flare that was managed by the gastroenterology team. Two weeks after hospitalization, the patient was discharged home in stable condition. As an outpatient, he received prednisolone with tapering instructions, azathioprine for his UC, levetiracetam for seizure, and warfarin with an INR goal of 2-3. The gastroenterology team also recommended infusion of infliximab after discharge. During a follow-up visit 8 weeks later, the patient's repeat MRI/MRV showed persistent compromise of the anterior aspect of the superior sagittal sinus, right transverse, and sigmoid sinuses. The posterior aspect of the superior sagittal sinus, transverse, and sigmoid sinuses were widely patent. The deep venous system was also patent, and the frontoparietal hemorrhage was resolved.

\section{Conclusion}

CVT is a rare manifestation of UC and is very challenging to diagnose and manage. It is crucial to do MRI/MRV for patients who present with headache and signs of increased intracranial pressure. Management and treatment of CVT involve a multidisciplinary team involving neurologist, gastroenterologist, radiologist, hematologist, neurosurgeon, intensive care, as well as physical and occupational therapists. It is crucial for clinicians treating UC patients presenting with neurological symptoms suggestive of a hemorrhagic brain lesion to have an index of suspicion for CVT in order to investigate the hypercoagulable states associated with this disease. Those patients who survive the initial impact of UC with CVT will need long-term follow-up with MRV and aggressive management of future UC flares.

\section{Statement of Ethics}

Written informed consent was obtained from the patient for publication of this case report and accompanying images.

\section{Conflict of Interest Statement}

The authors declare no conflict of interest.

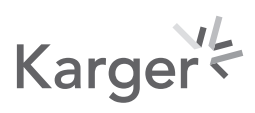


Mohamud et al.: Ulcerative Colitis and Sinus Vein Thrombosis

Funding Sources

The authors did not receive any funding.

\section{Author Contributions}

S.M., M.O., and R.W. managed the patient and collected clinical data. S.M. prepared the first draft. R.M.M. and O.D. edited and finalized the manuscript. All the authors reviewed and approved the final manuscript.

\section{References}

1 Bernstein CN, Blanchard JF, Houston DS, Wajda A. The incidence of deep venous thrombosis and pulmonary embolism among patients with inflammatory bowel disease: a population-based cohort study. Thromb Haemost. 2001;85(3):430-4.

2 Murthy SK, Nguyen GC. Venous thromboembolism in inflammatory bowel disease: an epidemiological review. Am J Gastroenterol. 2011;106(4):713-8.

3 Yuhara H, Steinmaus C, Corley D, Koike J, Igarashi M, Suzuki T, et al. Meta-analysis: the risk of venous thromboembolism in patients with inflammatory bowel disease. Aliment Pharmacol Ther. 2013;37(10):953-62.

4 Tan VP, Chung A, Yan BP, Gibson PR. Venous and arterial disease in inflammatory bowel disease. J Gastroenterol Hepatol. 2013;28(7):1095-113.

5 Tichelaar YI, Kluin-Nelemans HJ, Meijer K. Infections and inflammatory diseases as risk factors for venous thrombosis. A systematic review. Thromb Haemost. 2012;107(5):827-37.

6 Cho YH, Chae MK, Cha JM, Lee JI, Joo KR, Shin HP, et al. Cerebral venous thrombosis in a patient with Crohn's disease. Intest Res. 2016 Jan;14(1):96-101.

7 Lee J, Hwang SW, Lee J, Jung KH, Kim HI, Park SH, et al. A case of ulcerative colitis presenting with cerebral venous thrombosis. Intest Res. 2018;16(2):306-11.

8 Abdalla AO, Alluri D, Hassaballa M, Calvo L, Otaki F. A case of cerebral venous sinus thrombosis presenting during relapse of ulcerative colitis. Am J Case Rep. 2019;20:419-22.

9 Gunes HN, Cokal BG, Guler SK, Yoldas TK, Malkan UY, Demircan CS, et al. Clinical associations, biological risk factors and outcomes of cerebral venous sinus thrombosis. J Int Med Res. 2016;44(6):1454-61.

10 Mahale R, Mehta A, John AA, Buddaraju K, Shankar AK, Javali M, et al. Acute seizures in cerebral venous sinus thrombosis: what predicts it? Epilepsy Res. 2016;123:1-5.

11 Duman T, Uluduz D, Midi I, Bektas H, Kablan Y, Goksel BK, et al. A multicenter study of 1144 patients with cerebral venous thrombosis: the VENOST Study. J Stroke Cerebrovasc Dis. 2017 Aug;26(8):1848-57.

12 Komro J, Findakly D. Cerebral venous sinus thrombosis in adults with prothrombotic conditions: a systematic review and a case from our institution. Cureus. 2020;12(4):e7654.

13 Umit H, Asil T, Celik Y, Tezel A, Dokmeci G, Tuncbilek N, et al. Cerebral sinus thrombosis in patients with inflammatory bowel disease: a case report. World J Gastroenterol. 2005;11(34):5404-7.

14 Wang B, Peng C, Zhou MK, Guo J, He L. [Clinical characteristics and prognosis of patients with cerebral venous sinus thrombosis in southwest China]. Sichuan Da Xue Xue Bao Yi Xue Ban. 2014;45(3):515-8.

15 Sidhom Y, Mansour M, Messelmani M, Derbali H, Fekih-Mrissa N, Zaouali J, et al. Cerebral venous thrombosis: clinical features, risk factors, and long-term outcome in a Tunisian cohort. J Stroke Cerebrovasc Dis. 2014; 23(6):1291-5.

16 De Bruijn SF, de Haan RJ, Stam J. Clinical features and prognostic factors of cerebral venous sinus thrombosis in a prospective series of 59 patients. For The Cerebral Venous Sinus Thrombosis Study Group. J Neurol Neurosurg Psychiatry. 2001;70(1):105-8.

17 Algahtani HA, Abdu AP, Shami AM, Hassan AE, Madkour MA, Al-Ghamdi SM, et al. Cerebral venous sinus thrombosis in Saudi Arabia. Neurosciences. 2011 Oct;16(4):329-34.

18 Ferro JM, Canhão P, Bousser MG, Stam J, Barinagarrementeria F; ISCVT Investigators. Early seizures in cerebral vein and dural sinus thrombosis: risk factors and role of antiepileptics. Stroke. 2008;39(4):1152-8.

19 Karadas S, Milanlioglu A, Gönüllü H, Sayin R, Aydin MN. Cerebral venous sinus thrombosis presentation in emergency department in Van, Turkey. J Pak Med Assoc. 2014;64(4):370-4.

20 Stam J. Thrombosis of the cerebral veins and sinuses. N Engl J Med. 2005;352(17):1791-8.

21 Ferro JM, Canhão P, Stam J, Bousser MG, Barinagarrementeria F, ISCVT Investigators. Prognosis of cerebral vein and dural sinus thrombosis: results of the International Study on Cerebral Vein and Dural Sinus Thrombosis (ISCVT). Stroke. 2004 Mar;35(3):664-70.

22 Alvis-Miranda HR, Milena Castellar-Leones S, Alcala-Cerra G, Rafael Moscote-Salazar L. Cerebral sinus venous thrombosis. J Neurosci Rural Pract. 2013;4(4):427-38.

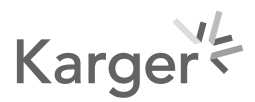

